\title{
Performance of Loop-Mediated Isothermal Amplification (LAMP)-Lateral Flow Detection and Xpert MTB/RIF Ultra for Diagnosis of Pulmonary Tuberculosis
}

\author{
Siripan Kounmee1, Uraporn Phumisantiphong'2, Yutthana Apichatbutr¹ \\ ${ }^{1}$ Pulmonology Division, Department of Internal Medicine, Faculty of Medicine, Vajira Hospital, Navamindradhiraj University, \\ Bangkok, Thailand \\ ${ }^{2}$ Department of Clinical Pathology, Central Laboratory and Blood Bank, Faculty of Medicine, Vajira Hospital, Navamindradhiraj \\ University, Bangkok, Thailand \\ Email:yutthana@nmu.ac.th
}

How to cite this paper: Kounmee, S., Phumisantiphong, U. and Apichatbutr, Y. (2021) Performance of Loop-Mediated Isothermal Amplification (LAMP)-Lateral Flow Detection and Xpert MTB/RIF Ultra for Diagnosis of Pulmonary Tuberculosis. Journal of Tuberculosis Research, 9, 245-255. https://doi.org/10.4236/jtr.2021.94023

Received: August 3, 2021

Accepted: November 7, 2021

Published: November 10, 2021

Copyright $\odot 2021$ by author(s) and Scientific Research Publishing Inc. This work is licensed under the Creative Commons Attribution-NonCommercial International License (CC BY-NC 4.0). http://creativecommons.org/licenses/by-nc/4.0/ (c) (i) (8) Open Access

\begin{abstract}
Background: Due to the limitations of diagnosis by Xpert MTB/RIF assay, WHO suggests Loop-Mediated Isothermal Amplification (TB-LAMP) instead of sputum-smear microscopy for pulmonary TB diagnosis in patients. Dr. Thongchai Kaewphinit et al. invented a fast TB detection kit called TB d-tect (LAMP-LFD assay). There was no clinical trial to estimate the performance of TB d-tect. Objective: This study was aimed to find the performance of LFD assay and Xpert MTB/RIF ultra for pulmonary TB. Material and methods: A cross-sectional study was conducted. Suggestive pulmonary TB patients were enrolled from June 2020-28 February 2021. Respiratory specimens were collected from each patient and sent for AFB smear, LAMP-LFD assay, Xpert MTB/RIF ultra and culture TB. Result: 139 patients with suspected pulmonary $\mathrm{TB}$ were enrolled. $51 \%$ of patients were diagnosed with pulmonary tuberculosis. Based on culture TB as a gold standard, the sensitivity and specificity of LAMP-LFD assay were $85.4 \%$ (95\% CI: 70.8\% - 94.4\%) and $87.8 \%$ (95\% CI: 79.6 - 93.5), respectively. The sensitivity and specificity of Xpert MTB/RIF ultra were 95.1\% (95\% CI: 83.5\% - 99.4\%) and 74.5\% (95\% CI: $64.7-82.8$ ), respectively. According to ROC curve, it was found that the areas under the curve of LAMP-LFD assay and Xpert MTB/RIF ultra were 0.866 and 0.848 , respectively $(\mathrm{p}=0.546$ ). Conclusion: The diagnostic sensitivity and specificity of LAMP-LFD assay appeared to be comparable to those of Xpert MTB/RIF ultra. LAMP-LFD assay could be used in resource limiting laboratory.
\end{abstract}


Keywords

LAMP-LFD Assay (TB d-Tect), Xpert MTB/RIF Ultra, Pulmonary TB

\section{Introduction}

Tuberculosis (TB) is an airborne transmitted disease caused by Mycobacterium tuberculosis. Most cases of TB are pulmonary TB; thus, it can be transmitted easily. The World Health Organization (WHO) ranked Thailand as the first among 14 top countries with the highest incidence of TB infection [1]. The key principle is to eradicate TB by preventing transmission. This means providing treatment early upon detection. Therefore, fast and accurate diagnosis is required. Thailand currently applies molecular biotechnology for pulmonary TB diagnosis based on the principle of polymerase chain reaction, e.g., Xpert MTB/ RIF, along with acid-fast staining (AFB) that has been used widely despite its low sensitivity. However, the Xpert MTB/RIF assay requires lab experts and fully equipped labs, and TB detection is expensive [2] and restricted to medical schools, medical centers, and regional disease control offices.

The newest version of Xpert MTB/RIF assay is the Xpert MTB/RIF ultra with diagnostic performance of $88 \%$ sensitivity and $96 \%$ specificity. Its overall performance is higher than that of Xpert MTB/RIF, with $85 \%$ sensitivity and $98 \%$ specificity [3]. This equipment is recommended by the WHO for the diagnosis of TB. As regards advantages, Xpert MTB/RIF ultra takes approximately $2 \mathrm{~h}$ to detect $\mathrm{TB}$ and provides primary data of rifampicin resistance. However, it ultra can only process $4-8$ specimens at a time, which is still insufficient for TB diagnosis in Thailand. Owing to the diagnostic limitations of the Xpert MTB/RIF assay, the WHO suggests the use of loop-mediated isothermal amplification (TBLAMP), instead of sputum-smear microscopy, for pulmonary TB diagnosis. The TB-LAMP assay is also suggested after smear microscopy, particularly in-patients with negative smear microscopy results; thus, relevant data must be collected, with three sputum collection standards. Based on references obtained from at least one sputum culture for TB (Standard 3), TB-LAMP will have 75.8\% (63.2 - 85.0) pooled sensitivity and $98.2 \%$ (96.0 - 99.2) pooled specificity [4].

In Thailand, Dr. Thongchai Kaewphinit and colleagues [5] invented a fast TB detection kit called TB d-tect. The principle of TB-LAMP is to increase the number of DNA by using four sets of primers specific to the IS6110 gene, which is specific to six sites of the target gene of $M$. tuberculosis, this detection method has high specificity. Interpretation of results is hastened up by reading through lateral flow dip stick (LFD) based on the principles of nucleic acid hybridization and chromatography. Therefore, LAMP-LFD assay is another suitable method for TB diagnosis for most labs of hospitals in Thailand with limited resources. It produces accurate and fast results $(<1 \mathrm{~h})$ and entails lower cost than the Xpert MTB/RIF assay. 
More studies have been conducted so far for pulmonary TB diagnosis by LAMP-LFD assay. Dr. Thongchai Kaewphinit and colleagues [6] conducted a sputum test (clinical unknown sample) using 101 specimens from the National Tuberculosis Reference Laboratory and found that TB diagnosis by LAMP-LFD had $98.92 \%$ sensitivity and $100 \%$ specificity, compared with culture examination.

This study aimed to assess the performance of LFD assay and Xpert MTB/RIF ultra to detect pulmonary TB by applying pulmonary TB diagnostic methods in accordance with methods used by most hospitals in Thailand under limited resource setting.

\section{Methods}

\subsection{Patients}

\section{Inclusion Criteria}

The participants must meet the following inclusion criteria:

1) Age $\geq 18$ years.

2) Patients with suspected symptoms of pulmonary $T B$, i.e., fever, chronic cough, weight loss, asymptomatic cases with CXR finding compatible with pulmonary TB.

Respiratory specimens could be collected.

\section{Exclusion Criteria}

Patients who have received TB treatment before, or have had pulmonary TB within 5 years.

\subsection{Objective}

\section{Primary Objective}

To detect sensitivity, specificity, and accuracy of LAMP-LFD assay to diagnose pulmonary TB.

\section{Secondary Objectives}

1) To detect sensitivity, specificity, and accuracy of LAMP-LFD assay to diagnose TB based on positive and negative AFB smears.

2) To examine agreement between results of LAMP-LFD assay and Xpert MTB/RIF ultra.

3) To find the relationship between chest X-ray (CXR) images and LAMP-LFD assay.

\subsection{Trial Design}

This is a cross-sectional study, approved by the Research Ethic Committee, Faculty of Medicine, Vajira Hospital. Sputum suspected of pulmonary TB would be brought for AFB and biomolecular detection by Xpert MTB/RIF ultra assay, LFD-LAMP assay, and sputum culture for TB in a lab with biosafety Level 2 . There were 3 methods with the following details.

1) The sputum stains for the Ziehl-Neelsen method were prepared and stain with $1 \%$ carbol fuchsin solution for 5 minutes, rinsed with water, and drained. 
They were decolorized with 3\% hydrochoric acid in alcohol for 5 minutes. Then slides were rinsed and counterstained with $0.1 \%$ methylene blue solution for 1 minute and rinsed with water. The slides were air-dried and examined using oil immersion objective (100x), red coloured acid fast bacilli were taken as positive and negative slides were those without any acid fast bacilli.

2) Xpert MTB/RIF ultra assay by dissolve sputum in sample reagent which contains sodium hydroxide (5\% - 8\%) and isopropyl alcohol (10\% - 15\%) with a volume of $1.5-2.0 \mathrm{ml}$. in a bottle with a sample of $0.5-1.0 \mathrm{ml}$. in ratio of 2:1 then, they were mixed with vortex mixture and left for 10 minutes under the room temperature, $2 \mathrm{ml}$ of mixed sample was put into the cartridge and start amplification of DNA, which take approximately 2 hours to analyze using Cepheid, Dx System Version 4.7b.

3) TB d-tect of Bioadvancetech Company was used for LAMP-LFD assay. This study used $200 \mu \mathrm{l}$ remained sputum from the preparation of Xpert MTB/RIF ultra then, put it to automated DNA extraction. Then, the extracted DNA was brought to increase the amount by LAMP technique under $61^{\circ} \mathrm{C}$ for 60 minutes. After that, $5 \mu \mathrm{l}$ of it was sucked out. $100 \mu \mathrm{l}$ assay buffer TB was added in it and mixed in the test tube. Next, the lateral flow dipstick was put into the tube and left for 10 minutes. Finally, the results were read. If the color code appeared on the control band and the test band, the results were positive. If the color code appeared on the control band only, the results were negative.

For sputum culture for TB, as a gold standard, it was conducted in the lab of Bangkok Pathology Laboratory in a form of liquid culture. Sputum specimens in the sterile container were submitted, stored under $2^{\circ} \mathrm{C}-8^{\circ} \mathrm{C}$ until they arrived at the lab.

TB is confirmed bacteriologically if the specimens were positive either by smear microscopy, C/S, or any other methods certified by WHO, e.g., Xpert MTB/RIF assay or line probe assay.

\subsection{Statistical Analysis}

According to a previous study [3], the Xpert MTB/RIF ultra assay had a sensitivity of $88 \%$. In the present study, the authors expected that LAMP-LFD will have a sensitivity of $88 \%$ or over, with $\pm 15 \%$ of errors. In 2018,2382 patients were screened, while 372 were diagnosed with pulmonary TB at Vajira Hospital. Therefore, the prevalence of pulmonary $\mathrm{TB}$ at Vajira Hospital was approximately $13.7 \%$. With a power of $80 \%$ and alpha of 0.05 , the study should include a sample of 19 patients:

$$
n=\frac{1.96^{2} \times 0.88 \times(1-0.88)}{0.15^{2}}=18.03=19
$$

Moreover, according to the prevalence of pulmonary TB at Vajira Hospital (13.7\%), the total sample should include at least $19 / 0.137=138.69=139$ patients.

Data were presented as numbers, percentage, mean, $\mathrm{SD} /$ median, and range. 
Differences between LFD-LAMP and Xpert MTB/RIF ultra were compared by sputum C/S test for TB as a gold standard. Sensitivity, specificity, negative predictive value, positive predictive value, and accuracy were detected by receiver operating characteristic (ROC) curve analysis. Agreement between LAMP-LFD assay and Xpert MTB/RIF ultra were estimated by Cohen's kappa statistic. The relationship between abnormal CXR images and LAMP-LFD assay was examined by Chi-squared or Fisher's exact test. A two-tailed p-value of $<0.05$ was considered significant. Data were analyzed by Stata version 15.1 (StataCorp, College Station, TX, USA).

\section{Result}

\subsection{Participants}

According to data collection from June 2020-2021, it results revealed totally 139 participants. $62 \%$ of them were male, aged 53 years on average. $69 \%$ did not have underlying diseases. $12 \%$ had HIV and $15 \%$ had diabetes. $63 \%$ had productive coughs, $60 \%$ had weight loss, and $49 \%$ had fever. $62 \%$ had reticulonodular infiltration $62 \%$ and $63 \%$ had air bronchogram (Table 1). According to sputum test, $51 \%$ of participants were diagnosed with TB, $3.6 \%$ were diagnosed with non-TB Mycobacterium (NTM), and $7.2 \%$ were diagnosed with bronchiectasis $7.2 \%$ (Table 2).

\subsection{Primary Outcomes}

LFD-LAMP and Xpert MTB/RIF ultra were compared with sputum C/S for TB (gold standard). For pulmonary TB analysis, the sensitivity and specificity of the LAMP-LFD assay were $85.4 \%$ (95\% CI 70.8\% - 94.4\%) and 87.8\% (95\% CI 79.6\% - 93.5\%), respectively, while the accuracy was $87.1 \%$. The sensitivity and specificity of Xpert MTB/RIF ultra were $95.1 \%$ (95\% CI 83.5\% - 99.4\%) and 74.5\% (95\% CI 64.7\% - 82.8\%), respectively, while the accuracy was $80.6 \%$ (Table 3 ). The sensitivity of LAMP-LFD assay and Xpert MTB/RIF ultra were higher than that of AFB smear. According to the ROC curve, the areas under the curve of the LAMP-LFD assay and Xpert MTB/RIF ultra were 0.866 and 0.848 , respectively $(\mathrm{p}=0.546)$. LAMP-LFD and Xpert MTB/RIF ultra had substantial agreement, with Cohen's kappa value of 0.749 (95\% CI 0.610 - 0.836).

\subsection{Secondary Outcomes}

When the specimens were analyzed separately according to AFB smear results, 40 were found positive for TB. For sputum test by Xpert MTB/RIF ultra, compared with the sputum $\mathrm{C} / \mathrm{S}$ for $\mathrm{TB}$, the sensitivity and specificity were 100\% (95 CI $88.1 \%-100 \%)$ and $36.4 \%(10.9 \%-69.2 \%)$, respectively. For the LFD-LAMP, the sensitivity and specificity were $96.6 \%(95 \%$ CI $82.2 \%-99.9 \%)$ and $54.5 \%$ (95\% CI 23.4\% - 83.3\%), respectively ( $\mathrm{p}=0.2451$ ) (Table 4 ).

In the analysis of 99 specimens with negative AFB smear, Xpert MTB/RIF ultra had sensitivity and specificity of $83.3 \%$ (95\% CI 51.6\% - 97.9\%) and $79.3 \%$ 
Table 1. Baseline characteristics.

\begin{tabular}{|c|c|}
\hline Characteristic & N 139 (\%) \\
\hline \multicolumn{2}{|l|}{ Gender } \\
\hline Male & $86(61.87)$ \\
\hline female & $53(38.13)$ \\
\hline Age & $53.51( \pm 17.02)$ \\
\hline BMI & $19.93( \pm 5.11)$ \\
\hline \multicolumn{2}{|l|}{ Underlying disease } \\
\hline HIV & $18(12.95)$ \\
\hline $\mathrm{DM}$ & 15 (10.79) \\
\hline CKD & $5(3.60)$ \\
\hline Steroid used & $8(5.76)$ \\
\hline No underlying & $96(69.06)$ \\
\hline \multicolumn{2}{|l|}{ Symptoms } \\
\hline Asymptomatic & $26(18.71)$ \\
\hline Chronic cough & $87(62.59)$ \\
\hline Weight loss & $58(41.73)$ \\
\hline Fever & $47(33.81)$ \\
\hline Dyspnea & $19(13.67)$ \\
\hline Hemoptysis & $6(4.32)$ \\
\hline \multicolumn{2}{|l|}{ CXR finding } \\
\hline Reticulonodular infiltration & $87(62.59)$ \\
\hline Air bronchogram & $62(44.60)$ \\
\hline Cavity & $18(12.95)$ \\
\hline Mass-like opacities & $7(5.04)$ \\
\hline Normal CXR & $3(2.16)$ \\
\hline
\end{tabular}

Table 2. Final diagnosis.

\begin{tabular}{ccccccccccc}
\hline & & \multicolumn{2}{c}{ AFB } & \multicolumn{2}{c}{ LFD-LAMP TB } & Xpert MTB/RIF ultra & Culture TB \\
\cline { 3 - 10 } Final diagnosis & Total 139 & negative & positive & negative & positive & negative & positive & $\begin{array}{c}\text { Negative } \\
\text { TB }\end{array}$ & $\begin{array}{c}\text { Positive } \\
\text { TB }\end{array}$ \\
\hline Pulmonary TB & $71(51.1)$ & $34(47.9)$ & $37(52.1)$ & $24(33.8)$ & $47(66.2)$ & $7(9.9)$ & $64(90.1)$ & $30(42.2)$ & $41(57.8)$ \\
Pulmonary NTM & $5(3.6)$ & $37(52.1)$ & $3(60.0)$ & $5(100.0)$ & $0(0)$ & $5(100.0)$ & $0(0)$ & $5(100.0)$ & $0(0)$ \\
malignancy & $9(6.5)$ & $9(100.0)$ & $0(0)$ & $9(100.0)$ & $0(0)$ & $9(100.0)$ & $0(0)$ & $9(100.0)$ & $0(0)$ \\
Pneumonia & $8(5.8)$ & $8(100.0)$ & $0(0)$ & $8(100.0)$ & $0(0)$ & $8(100.0)$ & $0(0)$ & $8(100.0)$ & $0(0)$ \\
Volume overload & $1(0.7)$ & $1(100.0)$ & $0(0)$ & $1(100.0)$ & $0(0)$ & $1(100.0)$ & $0(0)$ & $1(100.0)$ & $0(0)$ \\
Bronchiectasis & $10(7.2)$ & $10(100.0)$ & $0(0)$ & $10(100.0)$ & $0(0)$ & $10(100.0)$ & $0(0)$ & $10(100.0)$ & $0(0)$ \\
Other diagnosis & $35(15.1)$ & $35(100.0)$ & $0(0)$ & $35(100.0)$ & $0(0)$ & $35(100.0)$ & $0(0)$ & $35(100.0)$ & $0(0)$ \\
\hline
\end{tabular}


Table 3. Results of the sputum test by LAMP-LFD, Xpert MTB/RIF ultra, and AFB smear.

\begin{tabular}{|c|c|c|c|c|c|c|c|}
\hline \multirow{2}{*}{$\begin{array}{l}\text { Assay } \\
\text { (N139) }\end{array}$} & \multicolumn{2}{|c|}{ TB culture } & \multirow{2}{*}{$\begin{array}{c}\text { Sensitivity } \\
(\%)\end{array}$} & \multirow{2}{*}{$\begin{array}{l}\text { Specificity } \\
(\%)\end{array}$} & \multirow{2}{*}{$\begin{array}{l}\text { PPV } \\
(\%)\end{array}$} & \multirow{2}{*}{$\begin{array}{c}\text { NPV } \\
(\%)\end{array}$} & \multirow{2}{*}{$\begin{array}{c}\text { Accuracy } \\
(\%)\end{array}$} \\
\hline & positive & negative & & & & & \\
\hline LFD-LAMP TB positive & 35 & 12 & \multirow{2}{*}{$\begin{array}{c}85.4 \\
(70.8-94.4)\end{array}$} & \multirow{2}{*}{$\begin{array}{c}87.8 \\
(79.6-93.5)\end{array}$} & \multirow{2}{*}{$\begin{array}{c}74.5 \\
(59.7-86.1)\end{array}$} & \multirow{2}{*}{$\begin{array}{c}93.5 \\
(86.3-97.6)\end{array}$} & \multirow{2}{*}{87.1} \\
\hline LFD-LAMP TB negative & 6 & 86 & & & & & \\
\hline Xpert MTB/RIF ultrat positive & 39 & 25 & \multirow{2}{*}{$\begin{array}{c}95.1 \\
(83.5-99.4)\end{array}$} & \multirow{2}{*}{$\begin{array}{c}74.5 \\
(64.7-82.8)\end{array}$} & \multirow{2}{*}{$\begin{array}{c}60.9 \\
(47.9-72.9)\end{array}$} & \multirow{2}{*}{$\begin{array}{c}97.3 \\
(90.7-99.7)\end{array}$} & \multirow{2}{*}{80.6} \\
\hline Xpert MTB/RIF ultrat negative & 2 & 73 & & & & & \\
\hline AFB smaer positive & 29 & 11 & \multirow{2}{*}{$\begin{array}{c}70.7 \\
(54.5-83.9)\end{array}$} & \multirow{2}{*}{$\begin{array}{c}88.8 \\
(80.8-94.3)\end{array}$} & \multirow{2}{*}{$\begin{array}{c}72.5 \\
(56.1-85.4)\end{array}$} & \multirow{2}{*}{$\begin{array}{c}87.9 \\
(79.8-93.6)\end{array}$} & \multirow{2}{*}{83.4} \\
\hline AFB smaer negative & 12 & 87 & & & & & \\
\hline
\end{tabular}

Table 4. Subgroup analysis in positive AFB smear.

\begin{tabular}{|c|c|c|c|c|c|c|c|}
\hline \multirow{2}{*}{$\begin{array}{l}\text { Assay } \\
(\mathrm{N}=40)\end{array}$} & \multicolumn{2}{|c|}{ TB culture } & \multirow{2}{*}{$\begin{array}{c}\text { Sensitivity } \\
(\%)\end{array}$} & \multirow{2}{*}{$\begin{array}{c}\text { Specificity } \\
(\%)\end{array}$} & \multirow{2}{*}{$\begin{array}{r}\text { PPV } \\
(\%)\end{array}$} & \multirow{2}{*}{$\begin{array}{l}\text { NPV } \\
(\%)\end{array}$} & \multirow{2}{*}{$\begin{array}{c}\text { Accuracy } \\
(\%)\end{array}$} \\
\hline & positive & negative & & & & & \\
\hline LFD-LAMP TB positive & 28 & 5 & \multirow{2}{*}{$\begin{array}{c}96.6 \\
(82.2-99.9)\end{array}$} & \multirow{2}{*}{$\begin{array}{c}54.5 \\
(23.4-83.3)\end{array}$} & \multirow{2}{*}{$\begin{array}{c}84.8 \\
(68.1-94.9)\end{array}$} & \multirow{2}{*}{$\begin{array}{c}85.7 \\
(42.1-99.6)\end{array}$} & \multirow{2}{*}{85} \\
\hline LFD-LAMP TB negative & 1 & 6 & & & & & \\
\hline Xpert MTB/RIF ultrat positive & 29 & 7 & \multirow{2}{*}{$\begin{array}{c}100 \\
(88.1-100)\end{array}$} & \multirow{2}{*}{$\begin{array}{c}36.4 \\
(10.9-69.2)\end{array}$} & \multirow{2}{*}{$\begin{array}{c}80.6 \\
(64-91.8)\end{array}$} & \multirow{2}{*}{$\begin{array}{c}100 \\
(39.8-100)\end{array}$} & \multirow{2}{*}{82.5} \\
\hline Xpert MTB/RIF ultrat negative & 0 & 4 & & & & & \\
\hline
\end{tabular}

(95\% CI 69.3\% - 87.3\%), respectively. By contrast, LAMP-LFD assay had sensitivity and specificity of $58.3 \%$ (95\% CI $27.7 \%-84.8 \%)$ and 92 (95\% CI $84.1 \%$ $96.7 \%)$, respectively $(\mathrm{p}=0.361)$ (Table 5$)$.

When considering the relationship of CXR finding and LAMP-LFD assay, no CXR finding was significantly related to the positive results of the LAMP-LFD assay.

\section{Discussion}

This study aimed to evaluate the performance of molecular detection for sputum test by LAMP-LFD assay (TB d-tect). To diagnose TB, LAMP-LFD assay had $85.4 \%$ sensitivity, with better performance in the positive smear group, and had 96.6\% sensitivity. Results of previous studies confirmed the present findings. Joon et al. [2] conducted sputum tests on 107 specimens of patients with suspected pulmonary TB and found that the sdaA LAMP assay had high performance in diagnosing TB. Their results also agreed with the results of diagnosis by Xpert MTB/RIF assay, with sensitivity and specificity of $100 \%$ and $97.2 \%$, respectively.

Nagai et al. [7] performed a meta-analysis of the accuracy of TB diagnosis by the LAMP assay. They analyzed 26 studies and found that the LAMP assay had a sensitivity and specificity of $89.6 \%$ (95\% CI $85.6 \%-92.6 \%)$ and $94 \%$ (95\% CI 91\% - 96.1\%). Likewise, in Thailand, Phetsuksiri et al. [8] revealed that simultaneous DNA hybridization and LAMP integrated with LFD had high sensitivity 
Table 5. Subgroup analysis in negative AFB smear.

\begin{tabular}{|c|c|c|c|c|c|c|c|}
\hline \multirow{2}{*}{$\begin{array}{c}\text { Assay } \\
(\mathrm{N}=99)\end{array}$} & \multicolumn{2}{|c|}{ TB culture } & \multirow{2}{*}{$\begin{array}{c}\text { Sensitivity } \\
(\%)\end{array}$} & \multirow{2}{*}{$\begin{array}{c}\text { Specificity } \\
(\%)\end{array}$} & \multirow{2}{*}{$\begin{array}{l}\text { PPV } \\
(\%)\end{array}$} & \multirow{2}{*}{$\begin{array}{l}\text { NPV } \\
(\%)\end{array}$} & \multirow{2}{*}{$\begin{array}{c}\text { Accuracy } \\
(\%)\end{array}$} \\
\hline & positive & negative & & & & & \\
\hline LFD-LAMP TB positive & 7 & 7 & \multirow{2}{*}{$\begin{array}{c}58.3 \\
(27.7-84.8)\end{array}$} & \multirow{2}{*}{$\begin{array}{c}92 \\
(84.1-96.7)\end{array}$} & \multirow{2}{*}{$\begin{array}{c}50 \\
(23-77)\end{array}$} & \multirow{2}{*}{$\begin{array}{c}94.1 \\
(86.8-98.1)\end{array}$} & \multirow{2}{*}{87.8} \\
\hline LFD-LAMP TB negative & 5 & 80 & & & & & \\
\hline Xpert MTB/RIF ultrat positive & 10 & 18 & \multirow{2}{*}{$\begin{array}{c}83.3 \\
(51.6-97.9)\end{array}$} & \multirow{2}{*}{$\begin{array}{c}79.3 \\
(69.3-87.3)\end{array}$} & \multirow{2}{*}{$\begin{array}{c}35.7 \\
(18.6-55.9)\end{array}$} & \multirow{2}{*}{$\begin{array}{c}97.2 \\
(90.2-99.7)\end{array}$} & \multirow{2}{*}{79.8} \\
\hline Xpert MTB/RIF ultrat negative & 2 & 69 & & & & & \\
\hline
\end{tabular}

for detecting TB. When compared with the Xpert MTB/RIF assay using clinical samples, the sensitivity values were $82.1 \%$ (95\% CI 75.4\% - 88.98\%) and $86.9 \%$ (95\% CI 80.5\% - 90.8\%), respectively, similar to the results of this study.

In the subgroup analysis of the negative smear group, the LAMP-LFD assay produced lower results than the Xpert MTB/RIF ultra, as indicated by the very small amount of TB virus in the specimens. However, in the subgroup analysis of the positive smear group, LAMP-LFD produced satisfactory results close to Xpert MTB/RIF ultra. The results in this study agreed with those of Pham et al. [9] who found that TB-LAMP had better performance in diagnosing TB in the positive smear group ( $97.9 \%$ sensitivity)than in the negative smear group (46.6\% sensitivity). In Thailand, Toonkomdang et al. [10] found that the performance of in-house LAMP assay in the positive smear group (sensitivity 90.9\%) was higher than that in the negative smear group (sensitivity 16.7\%).

This study revealed that pulmonary TB diagnosis by LAMP-LFD assay had higher sensitivity than sputum smear. This method can be useful for hospitals that still lack Xpert MTB/RIF ultra because it is easy and can be conducted by available equipment in the labs. In addition, it can analyze larger specimens than Xpert MTB/RIF ultra. Therefore, diagnostic burdens in central hospital or disease control offices will be relieved. This method also confirms M. tuberculosis, instead of NTM, with positive AFB smear as well.

Moreover, this study found that sputum C/S for TB could produce only $57 \%$ of M. tuberculosis, resulting in lower specificity of LAMP-LFD assay and Xpert MTB/RIF ultra than other studies. This finding conformed to that of Toonkomdang et al. [10], who found that only $47 \%$ of positive TB cultures. This implied that without molecular test, diagnostic errors could occur. The finding that no TB virus was detected in the sputum C/S was possibly due to the quality of specimens. To clarify, the researchers instructed the volunteers to collect morning sputum on their own. The quality of the specimen collected was not evaluated before submission. Transportation could be another reason, that is, whether the sputum sample had been left for a long time after collection or was kept under proper temperatures before submission; TB virus might have been dead before arriving at the lab. Diagnosis by sputum C/S for TB is not only limited by time but also by low results of diagnosis in medical practice. This finding agreed with that of de Castro et al. [11] who found that sputum C/S for TB had sensitivity 
and specificity of $43.9 \%$ and $97.8 \%$, respectively, for the first submission of sputum. However, for the second submission, it obtained higher sensitivity and specificity, i.e., $51.6 \%$ and $96.3 \%$, respectively. The researchers viewed that TB diagnosis should be conducted in labs by combining multiple methods for the best accuracy. According to the ROC curve Figure 1 analysis of each method for TB diagnosis, the LAMP-LFD assay or Xpert MTB/RIF ultra, combined with AFB smear, had higher performance than using AFB smear alone. The prevalence of pulmonary $\mathrm{TB}$ in this study was higher than that in the general population (51\%), because most participants were receiving care from the TB clinic. Therefore, the chance of TB was already high.

This study revealed that pulmonary TB diagnosis by the LAMP-LFD assay (TB d-tect) had satisfactory performance similar to Xpert MTB/RIF ultra assay, with higher sensitivity than using AFB smear alone. Previously, Wang et al. [12] conducted a study in China and confirmed the ability of LAMP to diagnose TB, compared with sputum C/S for TB; in addition, they found that LAMP had high sensitivity (98.6\%) in the positive AFB smear group and lower sensitivity (67.7\%) in the AFB smear negative group, confirming the results in the present study. Therefore, LAMP appears to be a suitable alternative for TB diagnosis in most hospitals in Thailand that still lack Xpert MTB/RIF ultra. Its advantages are as follows:

1) M. tuberculosis can be confirmed rather than NTM, which might be falsely positive by AFB smear.

2) Easy to perform does not require extra equipment. The process takes $1 \mathrm{~h}$ (DNA extraction is not included). The results can be read with naked eyes.

3) Low cost.

4) It helps reduce burdens of central units for Xpert MTB/RIF ultra detection.

In conclusion, TB d-tect is an alternative for biomolecular diagnosis for pulmonary TB, particularly in hospitals with limited resources. To increase TB diagnosis, it can be used in hospitals without Xpert MTB/RIF ultra, which can finally lead to rapid treatment and less transmission.

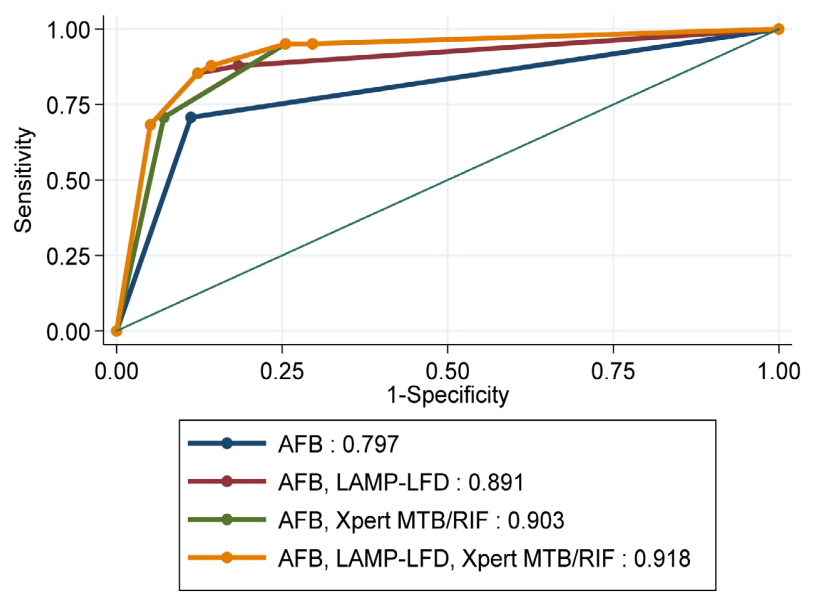

Figure 1. ROC curve for diagnosis of pulmonary TB with multiple test. 


\section{Acknowledgements}

This study received research fund from Navamindradhiraj University Research Fund and TB detection kits were received from Bioadvancetech Co., Ltd.

\section{Conflicts of Interest}

The authors declare no conflicts of interest regarding the publication of this paper.

\section{References}

[1] Imsanguan, W., Bupachat, S., Wanchaithanawong, V., Luangjina, S., Thawtheong, S., Nedsuwan, S., et al. (2020) Contact tracing for tuberculosis, Thailand. Bulletin of the World Health Organization, 98, 212-218.

https://doi.org/10.2471/BLT.19.239293

[2] Joon, D., Nimesh, M., Gupta, S., Kumar, C., Varma-Basil, M. and Saluja, D. (2019) Development and Evaluation of Rapid and Specific sdaA LAMP-LFD Assay with Xpert MTB/RIF Assay for Diagnosis of Tuberculosis. Journal of Microbiological Methods, 159, 161-166. https://doi.org/10.1016/j.mimet.2019.03.002

[3] Horne, D.J., Kohli, M., Zifodya, J.S., Schiller, I., Dendukuri, N., et al. (2019) Xpert MTB/RIF and Xpert MTB/RIF Ultra for Pulmonary Tuberculosis and Rifampicin Resistance in Adults. Cochrane Database of Systematic Reviews, 6, CD009593. https://doi.org/10.1002/14651858.CD009593.pub4

[4] World Health Organization (2016) The Use of Loop-Mediated Isothermal Amplification (TB-LAMP) for the Diagnosis of Pulmonary Tuberculosis: Policy Guidance.

[5] Kaewphinit, T., Ckumdee, J., Chansiri, K. and Santiwatanakul, S. (2017) Development and Evaluation of a Loop-Mediated Isothermal Amplification Combined with Au-Nanoprobe Assay for Rapid Detection of Mycobacterium tuberculosis. Indian Journal of Medical Microbiology, 35, 302-304. https://doi.org/10.4103/ijmm.IJMM $15 \quad 333$

[6] Kaewphinit, T., Arunrut, N., Kiatpathomchai, W., Santiwatanakul, S., Jaratsing, P. and Chansiri, K. (2013) Detection of Mycobacterium tuberculosis by Using LoopMediated Isothermal Amplification Combined with a Lateral Flow Dipstick in Clinical Samples. BioMed Research International, 2013, Article ID: 926230. https://doi.org/10.1155/2013/926230

[7] Nagai, K., Horita, N., Yamamoto, M., Tsukahara, T., Nagakura, H., Tashiro, K., et al. (2016) Diagnostic Test Accuracy of Loop-Mediated Isothermal Amplification Assay for Mycobacterium tuberculosis. Systematic Review and Meta-Analysis. Scientific Reports, 6, 1-10. https://doi.org/10.1038/srep39090

[8] Phetsuksiri, B., Klayut, W., Rudeeaneksin, J., Srisungngam, S., Bunchoo, S., Toonkomdang, S., et al. (2020) The Performance of an in-House Loop-Mediated Isothermal Amplification for the Rapid Detection of Mycobacterium tuberculosis in Sputum Samples in Comparison with Xpert MTB/RIF, Microscopy and Culture. Revista do Instituto de Medicina Tropical de São Paulo, 62, e36. https://doi.org/10.1590/s1678-9946202062036

[9] Pham, T.H., Peter, J., Mello, F.C.Q., Parraga, T., Lan, N.T.N., Nabeta, P., et al. (2018) Performance of the TB-LAMP Diagnostic Assay in Reference Laboratories: Results from a Multicentre Study. International Journal of Infectious Diseases, 68, 44-49. https://doi.org/10.1016/j.ijid.2018.01.005 
[10] Toonkomdang, S., Phinyo, P., Phetsuksiri, B., Patumanond, J., Rudeeaneksin, J. and Klayut, W. (2020) Pragmatic Accuracy of an in-House Loop-Mediated Isothermal Amplification (LAMP) for Diagnosis of Pulmonary Tuberculosis in a Thai Community Hospital. PloS One, 15, e0236496.

https://doi.org/10.1371/journal.pone.0236496

[11] e Castro, A.T., Mendes, M., Freitas, S. and Roxo, P. (2015) Diagnostic Yield of Sputum Microbiological Analysis in the Diagnosis of Pulmonary Tuberculosis in a Period of 10 Years. Revista Portuguesa de Pneumologia (English Edition), 21, 185-191. https://doi.org/10.1016/j.rppnen.2014.09.010

[12] Wang, Z.D., Sun, H.Y., Ren, Z.S., Xue, B., Lu, J. and Zhang, H.Q. (2019) Feasibility and Performance of Loop-Mediated Isothermal Amplification Assay in the Diagnosis of Pulmonary Tuberculosis in Decentralized Settings in Eastern China. BioMed Research International, 2019, Article ID: 6845756.

https://doi.org/10.1155/2019/6845756 